\title{
Chemical Compound Index
}

Acenaphthene $\left(\mathrm{C}_{12} \mathrm{H}_{10}\right) 105,106$, 205, 206, 430

Acenaphthyl $\left(\mathrm{c}-\mathrm{C}_{12} \mathrm{H}_{9}\right) 365$

Acenaphthylene $\left(\mathrm{C}_{12} \mathrm{H}_{18}\right)$ 83, 105 , 106, 206

Acetylene $\left(\mathrm{C}_{2} \mathrm{H}_{2}\right)$ 184, 185, 192, 193, 195, 196, 201, 205, 206, 237. 238, 279, 280

Acridine $\left(\mathrm{C}_{13} \mathrm{H}_{9} \mathrm{~N}\right) 429$

Ammonia $\left(\mathrm{NH}_{3}\right)$ 192, 305, 306, 307

Anthracene $\left(\mathrm{C}_{14} \mathrm{H}_{10}\right)$ 104-106, 253, 352, 356, 429, 430

Azulene $\left(\mathrm{C}_{10} \mathrm{H}_{8}\right)$ 105, 106, 363, 429, 430

Benzene $\left(\mathrm{C}_{6} \mathrm{H}_{6}\right)$ 104-106, 181, 182, 184, 186, 194, 227, 235, 236, 237, 238, 242ff, 279, 299, 363, 395, 430, 432

Benzo(g,h,i)perylene $\left(\mathrm{C}_{22} \mathrm{H}_{12}\right) 253$

Benzene, protonated $\left(\mathrm{C}_{6} \mathrm{H}_{7}^{+}\right) 66$, 103ff, 430

Benzyne $\left(\mathrm{C}_{6} \mathrm{H}_{4}\right) 193$

I,3 butendienyl $\left(1-\mathrm{C}_{4} \mathrm{H}_{5}\right) 182$

1-buten-3-ynyl $\left(\mathrm{l}^{-} \mathrm{C}_{3} \mathrm{H}_{3}\right) 182$

Carbon chain 70

Carbondioxide $\left(\mathrm{CO}_{2}\right)$ 306, 323

Carbon monoxide (CO) 185, 186 , 279, 305, 306, 307, 320

Carbon nanotube 113

Carbon sulfide (CS) 185, 192

$\mathrm{CH}_{5}^{+} 70$

$\mathrm{C}_{3}$ 299, 352, 365

$\mathrm{C}_{3} \mathrm{H}_{2} 355$

$\mathrm{C}_{3} \mathrm{H}_{4} 238$

$\mathrm{C}_{4} \mathrm{H} 192$

$\mathrm{C}_{4} \mathrm{H}_{3}^{+} 237,238$

$\mathrm{C}_{5} 352$

$\mathrm{C}_{6} \mathrm{H}^{-} 376$

$\mathrm{C}_{78} \mathrm{H}_{26} 362,363$

$\mathrm{C}_{96} \mathrm{H}_{30} 362,363$

$\mathrm{C}_{222} \mathrm{H}_{42}$ 295, 358, 362
Chrysene $\left(\mathrm{C}_{18} \mathrm{H}_{12}\right) 180$

Circumcoronene $\left(\mathrm{C}_{54} \mathrm{H}_{18}\right)$ 195, 443

Circumovalene $\left(\mathrm{C}_{66} \mathrm{H}_{20}\right)$ 127, 128

CN 192

Coranulene $\left(\mathrm{C}_{20} \mathrm{H}_{10}\right) 378$

Coronene $\left(\mathrm{C}_{24} \mathrm{H}_{12}\right)$ 61, 69, 104-106, 113, 114, 194, 195, 227, 233, 253, 357, 364, 376, 377, 395, 429, 430, 437, 448, 449, 450, 455, 457, 458

coronene, deprotonated $\left(\mathrm{C}_{24} \mathrm{H}_{11}^{-}\right) 376$

coronene, protonated $\left(\mathrm{C}_{24} \mathrm{H}_{13}^{+}\right) 375$

Cyclopropenylidene $\left(\mathrm{c}-\mathrm{C}_{3} \mathrm{H}_{2}\right) 235$

Diacetylene $\left(\mathrm{C}_{4} \mathrm{H}_{2}\right) 235,236$

Dicarbon radical $\left(\mathrm{C}_{2}\right)$ 187, 299, 344, 365

Dihydronaphthalene $\left(\mathrm{C}_{10} \mathrm{H}_{10}\right) 436$

Diamondoids 383

Ethane $\left(\mathrm{C}_{2} \mathrm{H}_{6}\right) 203,204$

Ethylene $\left(\mathrm{C}_{2} \mathrm{H}_{4}\right)$ 203, 204

Ethylene oxide $\left(\mathrm{c}-\mathrm{C}_{2} \mathrm{H}_{4} \mathrm{O}\right) 236$

Ethylnaphthalene $\left(\mathrm{C}_{22} \mathrm{H}_{18}\right) 239$

Ethynyl radical $\left(\mathrm{C}_{2} \mathrm{H}\right)$ 192, 193, 430

Fluoranthene $\left(\mathrm{C}_{16} \mathrm{H}_{10}\right)$ 83, 84, 429, 430

Fluorene $\left(\mathrm{C}_{13} \mathrm{H}_{10}\right) 356$

Formaldehyde $\left(\mathrm{H}_{2} \mathrm{CO}\right) 305$

Formamide $\left(\mathrm{NH}_{2} \mathrm{CHO}\right) 305$

Formyl ion $\left(\mathrm{HCO}^{+}\right) 238$

Fullerene ( $\mathrm{C}_{60}$ and others) 209ff, 297, 301, 302, 351, 353, 383, 429, 431, 432

Fulvene $\left(\mathrm{C}_{6} \mathrm{H}_{6}\right) 181$

Graphene 113

Graphite 30

$\mathrm{H}_{2}$ 244, 247, 276, 321, 322, 394, 419

$\mathrm{HC}_{4} \mathrm{H}^{+} 352$

$\mathrm{HC}_{11} \mathrm{~N} 305,361$

Hexa-peri-hexabenzocoronene $\left(\mathrm{C}_{42} \mathrm{H}_{18}\right)$ 362,374

Hydrogen cyanide (HCN) 185, 192, 320

Hydronaphthalene $\left(\mathrm{C}_{10} \mathrm{H}_{9}\right) 365$

Hydronaphthyl $\left(\mathrm{C}_{10} \mathrm{H}_{9}\right) 431$ 
Hydronium $\left(\mathrm{H}_{3} \mathrm{O}^{+}\right) 70$

Hydroxyl (OH) 186, 430

Indanyl $\left(\mathrm{C}_{9} \mathrm{H}_{9}\right) 365$

Indynyl $\left(\mathrm{C}_{9} \mathrm{H}_{7}\right) 365$

Methane $\left(\mathrm{CH}_{4}\right)$ 203, 204, 279, 280, 306,320

Methanol $\left(\mathrm{CH}_{3} \mathrm{OH}\right) 306,307$

Methyl $\left(\mathrm{CH}_{3}\right) 430$

Methylene $\left(\mathrm{CH}_{2}\right) 182$

Methylidyne (CH) 349, 353, 365, 430

Methylidyne cation $\left(\mathrm{CH}^{+}\right) 349$

Methylnaphthalene $\left(\mathrm{C}_{11} \mathrm{H}_{10}\right) 205$

Naphthalene $\left(\mathrm{C}_{10} \mathrm{H}_{8}\right) 64,65,66,69$, $81,82,86,88,104,106,126,127$, 203, 205, 231, 239, 352, 359, 363, $364,428,429,430,432,449$

Naphthalene, protonated $\left(\mathrm{C}_{10} \mathrm{H}_{9}^{+}\right)$ 104-106

Naphthylmethyl $\left(\mathrm{C}_{11} \mathrm{H}_{9}\right) 365$

Ovalene $\left(\mathrm{C}_{32} \mathrm{H}_{14}\right) 362$

Pentacene $\left(\mathrm{C}_{22} \mathrm{H}_{14}\right)$ 105, 106, 429

Perhydrocoronene $\left(\mathrm{C}_{24} \mathrm{H}_{36}\right) 457$

Perylene $\left(\mathrm{C}_{20} \mathrm{H}_{12}\right)$ 105, 106, 363, 429
Phenanthrene $\left(\mathrm{C}_{14} \mathrm{H}_{10}\right)$ 63, 180, 429, 430

Phenyl $\left(\mathrm{C}_{6} \mathrm{H}_{5}\right)$ 181, 186, 239

Phylpropargyl $\left(\mathrm{C}_{9} \mathrm{H}_{7}\right) 365$

Polyynes 182, 186, 187, 294, 301

Propargyl $\left(\mathrm{C}_{3} \mathrm{H}_{3}\right)$ 181, 182, 186, 193, 238

Propynylidene $\left(\mathrm{C}_{3} \mathrm{H}\right) 192$

Pyrene $\left(\mathrm{C}_{16} \mathrm{H}_{10}\right)$ 68, 69, 97, 98, 105, 106, 113, 127, 182, 193, 194, 195, 198, 252, 253, 312, 363, 428, 430, 432, 443

Pyrimidine $\left(\mathrm{C}_{4} \mathrm{H}_{4} \mathrm{~N}_{2}\right) 309$

Quaterrylene $\left(\mathrm{C}_{40} \mathrm{H}_{20}\right) 310$

Silicon monoxide ( $\mathrm{SiO}) 186$

Sulfur dioxide $\left(\mathrm{SO}_{2}\right) 323$

Terrylene $\left(\mathrm{C}_{30} \mathrm{H}_{16}\right) 362$

Tetracene $\left(\mathrm{C}_{18} \mathrm{H}_{12}\right)$ 105, 106, 429

Triacetylene $\left(\mathrm{C}_{6} \mathrm{H}_{2}\right)$ 235, 236

Trihydronaphthalene $\left(\mathrm{C}_{10} \mathrm{H}_{11}\right) 365$

Triphenylene $\left(\mathrm{C}_{18} \mathrm{H}_{12}\right) 362,429$

Uracil $\left(\mathrm{C}_{4} \mathrm{H}_{2} \mathrm{~N}_{2} \mathrm{O}_{2}\right) 309$

Vinylacetylene $\left(\mathrm{C}_{4} \mathrm{H}_{4}\right) 182$

Water $\left(\mathrm{H}_{2} \mathrm{O}\right)$ 186, 306, 307, 320, 323 